\title{
On Common Fixed Point Theorems in the Stationary Fuzzy Metric Space of the Bounded Closed Sets
}

\author{
Dong Qiu, Chongxia Lu, Wei Zhang, and Yuzhong Mo \\ College of Mathematics and Physics, Chongqing University of Posts and Telecommunications, Nanan, Chongqing 400065, China \\ Correspondence should be addressed to Dong Qiu; dongqiumath@163.com
}

Received 11 July 2013; Accepted 9 September 2013

Academic Editor: Hassen Aydi

Copyright (C) 2013 Dong Qiu et al. This is an open access article distributed under the Creative Commons Attribution License, which permits unrestricted use, distribution, and reproduction in any medium, provided the original work is properly cited.

Under the $\phi$-contraction conditions, we prove common fixed point theorems for self-mappings in the space $\mathscr{C} \mathscr{B}(X)$ of the bounded closed sets in the complete stationary fuzzy metric space with the $H_{M}$-fuzzy metric for the bounded closed sets.

\section{Introduction}

It is well known that not only the Hausdorff metric is very important concept in general topology and analysis, but also many authors have expansively developed the theory of fuzzy sets and application (see [1-11]). As a natural generalization of the concept of set, fuzzy sets were introduced initially by Zadeh [12] in 1965. Various concepts of the fuzzy metrics on ordinary set were considered in [13-19].

In [20], Rodríguez-López and Romaguera introduced and discussed a suitable notion for the Hausdorff fuzzy metric of a given fuzzy metric space (in the sense of George and Veeramani) on the set of its nonempty compact subsets. It is necessary to note that such fuzzy metric space has very important application in studying fixed point theorems for contraction-type mappings [21-30]. In fuzzy functional analysis, many researches have been done on the fixed point theory in the space of compact fuzzy sets equipped with the supremum metric $[1,16,31-38]$.

In this paper, we will establish the completeness of $\left(\mathscr{C} \mathscr{B}(X), H_{M}, *\right)$ with respect to the completeness of the stationary fuzzy metric space $(X, M, *)$, where $\mathscr{C} \mathscr{B}(X)$ is the class of sets with nonempty bounded closed subsets of $X$, and $H_{M}$ is the stationary Hausdorff fuzzy metric on $\mathscr{C} \mathscr{B}(X)$ induced by $M$. Finally, we will prove some common fixed point theorems for self-mappings in the space $\mathscr{C} \mathscr{B}(X)$.

\section{Preliminaries}

We start this section by recalling some pertinent concepts.

Definition 1 (see [39]). A triangular norm (or $t$-norm for short) is a binary operation $*$ on the unit interval $[0,1]$, that is, a function $*:[0,1]^{2} \rightarrow[0,1]$, such that for all $a, b, c, d \in$ $[0,1]$ the following four axioms are satisfied:

(1) $a * 1=a$ (boundary condition);

(2) $a * b \leq c * d$ whenever $a \leq c$ and $b \leq d$ (monotonicity);

(3) $a * b=b * a$ (commutativity);

(4) $a *(b * c)=(a * b) * c$ (associativity).

A $t$-norm $*$ is said to be continuous if it is a continuous function in $[0,1]^{2}$; a $t$-norm $*$ is said to be positive if $a * b>0$ whenever $a, b \in(0,1]$. The following are examples of $t$ norms: $a *_{p} b=a \cdot b ; a \wedge b=\min (a, b)$, where $a \cdot b$ denotes the usual multiplication for all $a, b \in[0,1]$.

Definition 2 (see [40]). A stationary fuzzy metric space is an ordered triple $(X, M, *)$ such that $X$ is an arbitrary nonempty set, $*$ is a continuous $t$-norm, and $M$ is a fuzzy set of $X \times X$ satisfying the following conditions, for all $x, y, z \in X$ :

(1) $M(x, y)>0$,

(2) $M(x, y)=1$ if and only if $x=y$, 
(3) $M(x, y)=M(y, x)$,

(4) $M(x, y) \geq M(x, z) * M(z, y)$.

If $(X, M, *)$ is a stationary fuzzy metric space, we will say that $(M, *)$ is a stationary fuzzy metric on $X$. Since a stationary fuzzy metric is a special fuzzy metric, just like fuzzy metrics in [14], we can prove that every stationary fuzzy metric $(M, *)$ on $X$ generates a topology $\tau_{M}$ on $X$ which has as a base the family of sets of the form $\left\{B_{M}(x, \varepsilon): x \in X, 0<\varepsilon<1\right\}$, where $B_{M}(x, \varepsilon)=\{y \in X: M(x, y)>1-\varepsilon\}$ for all $\varepsilon \in(0,1)$. A sequence $\left(x_{i}\right)_{i \in \mathbb{N}}$ in a stationary fuzzy metric space $(X, M)$ is said to be Cauchy if $\lim _{i, j \rightarrow \infty} M\left(x_{i}, x_{j}\right)=1$; a sequence $\left(x_{i}\right)_{i \in \mathbb{N}}$ in $X$ converges to $x$ if $\lim _{i \rightarrow \infty} M\left(x_{i}, x\right)=1[40]$.

Example 3 (see $[14])$. Let $(X, d)$ be a metric space. Denote by $a \cdot b$ the usual multiplication for all $a, b \in[0,1]$, and define $M_{d}$ on $X \times X$ by

$$
M_{d}(x, y)=\frac{1}{1+d(x, y)}
$$

for all $x, y \in X$. Then $\left(M_{d}, \cdot\right)$ is a stationary fuzzy metric on $X$ which will be called a standard stationary fuzzy metric.

Definition 4 (see [41]). A stationary fuzzy pseudometric space is an ordered triple $(X, M, *)$ such that $X$ is an arbitrary nonempty set, $*$ is a continuous $t$-norm, and $M$ is a fuzzy set of $X \times X$ satisfying the following conditions, for all $x, y, z \in X$ :

(1) $M(x, y)=1$ if and only if $x=y$,

(2) $M(x, y)=M(y, x)$,

(3) $M(x, y) \geq M(x, z) * M(z, y)$.

If $(X, M, *)$ is a stationary fuzzy pseudometric space, we will say that $(M, *)$ is a stationary fuzzy pseudometric on $X$. In the following we always suppose that the $t$-norm $*$ is positive.

Definition 5 (see [42]). Let $(X, M, *)$ be a stationary fuzzy metric space and $A \subset X$. If, for all $\varepsilon \in(0,1), B_{M}(x, \varepsilon) \bigcap(A-$ $\{x\}) \neq \emptyset$, then $x$ is an accumulation point of $A$; the set of all accumulation points of $A$ is called the derived set of $A$, denoted by $d(A)$; the union of $A$ and $d(A)$ is called the closure of $A$, denoted by $\bar{A}$. If $d(A) \subset A$, then $A$ is a closed set of $X$.

Definition 6 (see [42]). Let $(X, M, *)$ be a stationary fuzzy metric space, and $A \subset X$. If there exists $r \in(0,1)$ such that for all $x, y \in A$ we have $M(x, y)>1-r$, then we say that $A$ is a bounded subset of $X$; if $X$ itself is a bounded set we will say that $(X, M, *)$ is a bounded stationary fuzzy metric space.

Given a stationary fuzzy metric space $(X, M, *)$, we will denote by $\mathscr{P}(X), \mathscr{P}_{0}(X)$, and $\mathscr{C} \mathscr{B}(X)$, the powerset, the set of nonempty subsets, and the set of nonempty bounded closed subsets of $(X, M, *)$, respectively.

Let $B$ be a nonempty subset of a stationary fuzzy metric space $(X, M, *)$. For all $x \in X$, let

$$
M(x, B)=\sup _{y \in B} M(x, y)=M(B, x) .
$$

For the empty index set $\emptyset$, we will make the convention that, for $a_{x} \in[0,1]$,

$$
\sup _{x \in \emptyset} a_{x}=0, \quad \inf _{x \in \emptyset} a_{x}=1 .
$$

It follows that $M(x, \emptyset)=M(\emptyset, x)=0$.

Definition 7 (see [43]). Let $(X, M, *)$ be a stationary fuzzy metric space. For all $A, B \in \mathscr{P}(X)$, we define a function $H_{M}$ on $\mathscr{P}(X) \times \mathscr{P}(X)$ by

$$
\begin{aligned}
H_{M}(A, B) & =\inf _{x \in A} M(x, B) \wedge \inf _{y \in B} M(y, A) \\
& =\rho(A, B) \wedge \rho(B, A)
\end{aligned}
$$

where $\rho(A, B)=\inf _{x \in A} M(x, B)$.

Definition 8 . Let $(X, M, *)$ be any stationary metric space. $A_{0} \in \mathscr{C} \mathscr{B}(X)$ is said to be a fixed point of a self-mapping $f$ of $\mathscr{C} \mathscr{B}(X)$ if and only if $A_{0} \subseteq f\left(A_{0}\right)$.

\section{Main Results}

Now we will establish our main theorems.

Proposition 9. Let $(X, M, *)$ be a stationary fuzzy metric space. Then, for all $A, B, C \in \mathscr{P}(X)$,

(1) $\rho(A, B)=1$ if and only if $A \subset \bar{B}$ if and only if $\bar{A} \subset \bar{B}$ if and only if $\rho(\bar{A}, \bar{B})=1$,

(2) for all $x, x^{\prime} \in X, M(x, B) \geq M\left(x, x^{\prime}\right) * M\left(x^{\prime}, B\right)$,

(3) $\rho(A, B)=\rho(A, \bar{B})=\rho(\bar{A}, \bar{B})$,

(4) $\rho(A, C) \geq \rho(A, B) * \rho(B, C)$,

(5) if $A \subset \bar{B}$, then $\rho(A, C) \geq H_{M}(B, C)$,

(6) $\rho(A, C) \geq H_{M}(A, B) * \rho(B, C)$,

(7) $H_{M}(A, B)=1$ if and only if $\bar{A}=\bar{B}$.

Proof. In fact, we can prove this proposition by a similar proof of Proposition 1 in [42].

Theorem 10. Let $(X, M, *)$ be a stationary fuzzy metric space, and then $\left(\mathscr{P}(X), H_{M}\right.$,*) is a stationary fuzzy pseudometric space.

Proof. Let $A, B, C \in \mathscr{P}(X)$; by the definition of $H_{M}$, (1) of Proposition 9, and the commutativity of $*$, it is clear that $H_{M}(A, B)=1$ if and only if $A=B$ and $H_{M}(A, B)=$ $H_{M}(B, A)$.

In addition, by (4) of Proposition 9 and the commutativity of $*$, we obtain

$$
\begin{aligned}
& \rho(A, C) \wedge \rho(C, A) \\
& \quad \geq(\rho(A, B) * \rho(B, C)) \wedge(\rho(C, B) * \rho(B, A)) \\
& \quad \geq(\rho(A, B) \wedge \rho(B, A)) *(\rho(B, C) \wedge \rho(C, B)) .
\end{aligned}
$$


Consequently, by the definition of $H_{M}$, we get

$$
H_{M}(A, C) \geq H_{M}(A, B) * H_{M}(B, C) .
$$

We conclude that $\left(\mathscr{P}(X), H_{M}\right.$, *) is a stationary fuzzy pseudometric space.

Proposition 11 (see [42]). Let $(X, M, *)$ be a stationary fuzzy metric space. If $A, B \subset X$ are any two bounded subsets of $X$, then $A \cup B$ is a bounded subset of $X$.

Theorem 12. Let $(X, M, *)$ be a stationary fuzzy metric space. Then $\left(\mathscr{C} \mathscr{B}(X), H_{M}, *\right)$ is a stationary fuzzy metric space.

Proof. Let $A, B, C \in \mathscr{C} \mathscr{B}(X)$. By Proposition 11, we have $A \cup$ $B \in \mathscr{C} \mathscr{B}(X)$, which means there exists $r \in(0,1)$ such that $M(x, y)>1-r$, for all $x \in A, y \in B$. Hence, for any $x \in A$, we can get that

$$
M(x, B)=\sup _{y \in B} M(x, y)>1-r>0 .
$$

Thus we obtain

$$
\rho(A, B)=\inf _{x \in A} M(x, B) \geq(1-r)>0 .
$$

Similarly, we can get

$$
\rho(B, A)=\inf _{y \in A} M(y, A) \geq(1-r)>0 .
$$

Consequently, by the positivity of $*$, we have $H_{M}(A, B)=$ $\rho(A, B) * \rho(B, A)>0$.

By the definition of $H_{M}$, (7) of Proposition 9, and the commutativity of $*$, it is clear that $H_{M}(A, B)=1$ if and only if $A=B$ and $H_{M}(A, B)=H_{M}(B, A)$. In addition, by (4) of Proposition 9 and the commutativity of $*$, we obtain

$$
\begin{aligned}
& \rho(A, C) \wedge \rho(C, A) \\
& \quad \geq(\rho(A, B) * \rho(B, C)) \wedge(\rho(C, B) * \rho(B, A)) \\
& \quad \geq(\rho(A, B) \wedge \rho(B, A)) *(\rho(B, C) \wedge \rho(C, B)) .
\end{aligned}
$$

Consequently, by the definition of $H_{M}$, we get

$$
H_{M}(A, C) \geq H_{M}(A, B) * H_{M}(B, C) .
$$

We conclude that $\left(\mathscr{C} \mathscr{B}(X), H_{M}, *\right)$ is a stationary fuzzy metric space.

Example 13. Let $(X, d)$ be a metric space. Then the Hausdorff stationary fuzzy metric $\left(H_{M_{d}}, \cdot\right)$ of the standard fuzzy metric $\left(M_{d}, \cdot\right)$ coincides with the standard fuzzy metric $\left(M_{H_{d}}, \cdot\right)$ of the Hausdorff fuzzy metric $H_{d}$ on $\mathscr{C} \mathscr{B}(X)$.

In fact, let $A, B \in \mathscr{C} \mathscr{B}(X)$; for each $x_{0} \in A$, we have

$$
\begin{aligned}
M_{d}\left(x_{0}, B\right) & =\sup _{y \in B} M_{d}\left(x_{0}, y\right)=\sup _{y \in B} \frac{1}{1+d\left(x_{0}, y\right)} \\
& =\frac{1}{1+\inf _{y \in B} d\left(x_{0}, y\right)}=\frac{1}{1+d\left(x_{0}, B\right)} .
\end{aligned}
$$

Consequently, we obtain

$$
\begin{aligned}
H_{M_{d}}(A, B) & =\inf _{x \in A} M_{d}(x, B) \wedge \inf _{y \in B} M_{d}(y, A) \\
& =\frac{1}{1+\sup _{x \in A} d(x, B)} \wedge \frac{1}{1+\sup _{y \in B} d(y, A)} \\
& =\frac{1}{1+\sup _{x \in A} d(x, B) \vee \sup _{y \in B} d(y, A)} \\
& =\frac{1}{1+H_{d}(A, B)}=M_{H_{d}}(A, B) .
\end{aligned}
$$

We conclude that $H_{M_{d}}=M_{H_{d}}$ on $\mathscr{C} \mathscr{B}(X)$.

Let us recall that if $(X, \mathcal{U})$ is a uniform space, then the Hausdorff-Bourbaki uniformity $H_{\mathscr{U}}$ (of $\mathcal{U}$ ) on $\mathscr{P}(X)$ has as a base the family of sets of the form

$$
H_{U}=\{(A, B) \in \mathscr{P}(X) \times \mathscr{P}(X): B \subseteq U(A), A \subseteq U(B)\},
$$

where $U \in \mathcal{U}[44]$.

The restriction of $H_{\mathscr{U}}$ to $\mathscr{C} \mathscr{B}(X) \times \mathscr{C} \mathscr{B}(X)$ will be also denoted by $H_{\mathscr{U}}$. In addition, if $(X, M, *)$ is a stationary fuzzy metric space, then $\left\{U_{n}: n \in \mathbb{N}^{+}\right\}$is a (countable) base for the uniformity $\mathcal{U}_{M}$ on $X$ compatible with $\tau_{M}$, where

$$
U_{n}=\left\{(x, y) \in X \times X: M(x, y)>1-\frac{1}{n}\right\}
$$

for all $n \in \mathbb{N}^{+} . \mathscr{U}_{M}$ is called the uniformity induced by $(M, *)$. In particular, $\mathscr{U}_{H_{M}}$ is the uniformity induced by the Hausdorff stationary fuzzy metric of $(M, *)$. We have the following useful result.

Theorem 14. Let $(X, M, *)$ be a stationary fuzzy metric space. Then the Hausdorff-Bourbaki uniformity $H_{\mathscr{U}_{M}}$ coincides with the uniformity $\mathcal{U}_{H_{M}}$ on $\mathscr{C} \mathscr{B}(X)$.

Proof. If $(A, B) \in\{(A, B) \in \mathscr{C} \mathscr{B}(X) \times \mathscr{C} \mathscr{B}(X): B \subseteq$ $\left.U_{n+1}(A), A \subseteq U_{n+1}(B)\right\}$, then, for any $y \in B \subseteq U_{n+1}(A)$, there exists $x_{y} \in A \subseteq U_{n+1}(B)$, such that $M\left(x_{y}, y\right)>1-$ $(1 /(n+1))$. Thus we obtain

$$
M(A, y)=\sup _{x \in A} M(x, y) \geq M\left(x_{y}, y\right)>1-\frac{1}{n+1},
$$

for all $y \in B \subseteq U_{n+1}(A)$. Consequently we have

$$
\inf _{y \in B} M(A, y) \geq 1-\frac{1}{n+1} \text {. }
$$

Similarly, we can get

$$
\inf _{x \in A} M(x, B) \geq 1-\frac{1}{n+1},
$$

for all $A \subseteq U_{n+1}(B)$. Thus we conclude that

$$
H_{M}(A, B)=\inf _{x \in A} M(x, B) \wedge \inf _{y \in B} M(A, y) \geq 1-\frac{1}{n+1} .
$$


If $(A, B) \in\left\{(A, B) \in \mathscr{C} \mathscr{B}(X) \times \mathscr{C} \mathscr{B}(X): H_{M}(A, B)>\right.$ $1-(1 / n)\}$, then, for each $x \in A$, we have

$$
M(x, B)=\sup _{y \in B} M(x, y)>1-\frac{1}{n} .
$$

Let $\alpha=M(x, B)$, and then, for each $x \in A$, there exists $x_{y} \in B$ such that

$$
\begin{aligned}
M\left(x, x_{y}\right) & \geq M(x, B)-\frac{\alpha+1 / n-1}{2}=\frac{\alpha-1 / n+1}{2} \\
& >1-\frac{1}{n} .
\end{aligned}
$$

Thus we obtain $A \subseteq U_{n}(B)$.

Similarly, by

$$
M(A, y)=\sup _{x \in A} M(x, y)>1-\frac{1}{n},
$$

we can get $B \subseteq U_{n}(A)$. It follows that

$$
\begin{aligned}
& (A, B) \\
& \in\left\{(A, B) \in \mathscr{C} \mathscr{B}(X) \times \mathscr{C} \mathscr{B}(X): B \subseteq U_{n}(A), A \subseteq U_{n}(B)\right\} .
\end{aligned}
$$

Hence we obtain the following relations:

$$
\begin{aligned}
\in & \left\{(A, B) \in \mathscr{C} \mathscr{B}(X) \times \mathscr{C} \mathscr{B}(X): B \subseteq U_{n+1}(A),\right. \\
& \left.A \subseteq U_{n+1}(B)\right\} \\
\subseteq & \left\{(A, B) \in \mathscr{C} \mathscr{B}(X) \times \mathscr{C} \mathscr{B}(X): H_{M}(A, B) \geq 1-\frac{1}{n+1}\right\} \\
\subseteq & \left\{(A, B) \in \mathscr{C} \mathscr{B}(X) \times \mathscr{C} \mathscr{B}(X): H_{M}(A, B)>1-\frac{1}{n}\right\} \\
\subseteq & (A, B) \\
\in & \left\{(A, B) \in \mathscr{C} \mathscr{B}(X) \times \mathscr{C} \mathscr{B}(X): B \subseteq U_{n}(A), A \subseteq U_{n}(B)\right\} .
\end{aligned}
$$

$$
\text { We conclude that } H_{U_{M}}=\mathscr{U}_{H_{M}} \text { on } \mathscr{C} \mathscr{B}(X) \text {. }
$$

Theorem 15. Let $(X, M, *)$ be a stationary fuzzy metric space. Then $\left(\mathscr{C} \mathscr{B}(X), H_{M}, *\right)$ is complete if and only if $(X, M, *)$ is complete.

Proof. In fact, we can prove it by a similar proof of Theorem 3 in [20].

Another type of convergence for a sequence of sets was defined by Kuratowski.

We say that a sequence of sets $\left\{C_{k}\right\}_{k=1}^{\infty}, C_{k} \subseteq X$, converges to a set $C \subseteq X$, denoted by $\lim _{k \rightarrow \infty} C_{k}=C$, if

$$
C=\lim \inf C_{k}=\lim \sup C_{k}
$$

where $\liminf C_{k}=\left\{x \in X: x=\lim _{k \rightarrow \infty} x_{k}, x_{k} \in C_{k}\right\}$, $\limsup C_{k}=\bigcap_{k=1}^{\infty}\left(\overline{\bigcup_{i=k}^{\infty} C_{i}}\right)$.
We mention that, for sequence of closed sets, convergence in Hausdorff metric implies convergence in the sense of Kuratoski. But for sequence of bounded closed sets, both types of convergence are equivalent provided that the limit set is nonempty [37].

Lemma 16. Let $(X, M, *)$ be a stationary fuzzy metric space and $A, B \in \mathscr{C} \mathscr{B}(X)$. Then

(1) for arbitrarily $\varepsilon \in(0,1)$ and any $x \in A$, there exists $y \in B$ such that $M(x, y) \geq H_{M}(A, B)-\varepsilon ;$

(2) for any $x \in A$ and any $\beta \in[0,1)$, there exists $y \in B$ such that $M(x, y) \geq \beta H_{M}(A, B)$.

Proof. We only prove (1) since it is equivalent to (2).

For each $x \in A$, there exists $y \in B$ such that, for any $\varepsilon \in(0,1)$,

$$
\begin{aligned}
M(x, y) & \geq \sup _{y \in B} M(x, y)-\varepsilon \geq \inf _{x \in A} \sup _{y \in B} M(x, y)-\varepsilon \\
& =\rho(A, B)-\varepsilon \geq \rho(A, B) \wedge \rho(B, A)-\varepsilon \\
& =H_{M}(A, B)-\varepsilon .
\end{aligned}
$$

This completes the proof.

In fact, we can get a more general result.

Lemma 17. Let $(X, M, *)$ be a stationary fuzzy metric space and $A, B \in \mathscr{C} \mathscr{B}(X)$. Then

(1) for arbitrarily $\varepsilon \in(0,1)$ and any closed subset $A_{1} \subseteq A$, there exists closed subset $B_{1} \subseteq B$ such that $H_{M}\left(A_{1}\right.$, $\left.B_{1}\right) \geq H_{M}(A, B)-\varepsilon$;

(2) for any closed subset $A_{1} \subseteq A$ and any $\beta \in[0,1)$, there exists closed subset $B_{1} \subseteq B$ such that $H_{M}\left(A_{1}, B_{1}\right) \geq$ $\beta H_{M}(A, B)$.

Proof. We only prove (1) since it is equivalent to (2). Let $\varepsilon \in$ $(0,1)$ and let

$$
\begin{aligned}
C_{0}=\left\{y: \text { there exists an } x \in A_{1}\right. \\
\left.\quad \text { such that } M(x, y) \geq H_{M}(A, B)-\varepsilon\right\} .
\end{aligned}
$$

Assume $z \in \overline{C_{0}}$. For any $\delta>0$, there exists a $y \in C_{0}$ such that $M(z, y)>1-\delta$. By (2) of Proposition 9, we have

$$
\begin{aligned}
M\left(z, A_{1}\right) & \geq M(z, y) * M\left(y, A_{1}\right) \\
& \geq(1-\delta) *\left(H_{M}(A, B)-\varepsilon\right) .
\end{aligned}
$$

By the arbitrariness of $\delta$, we have $M\left(z, A_{1}\right) \geq H_{M}(A, B)-\varepsilon$. Then we get

$$
\overline{C_{0}} \subseteq D=\left\{z: M\left(z, A_{1}\right) \geq H_{M}(A, B)-\varepsilon\right\} .
$$

Conversely, suppose $z \in D$. Take a descending positive number sequence $\left\{\delta_{n}\right\}_{n=1}^{\infty} \subseteq(0,1)$ such that $\delta_{n} \rightarrow 0$ as $n \rightarrow$ $\infty$. For each $\delta_{n}$, there exists a $x \in A_{1}$ such that

$$
M(z, x) \geq\left(1-\delta_{n}\right) *\left(H_{M}(A, B)-\varepsilon\right) .
$$


Let $C_{\delta_{n}}=\left\{y\right.$ : there exists an $x \in A_{1}$ such that $M(x$, $\left.y) \geq\left(1-\delta_{n}\right) *\left(H_{M}(A, B)-\varepsilon\right)\right\}$. Then we have $z \in C_{\delta_{n}} \subseteq$ $\overline{C_{\delta_{n}}}, n=1,2, \ldots$, and $\overline{C_{\delta_{i}}} \subseteq \overline{C_{\delta_{j}}}(i \geq j)$. Hence we can get

$$
z \in \lim _{n \rightarrow \infty} C_{\delta_{n}}=\bigcap_{n=1}^{\infty} \overline{C_{\delta_{n}}}=\overline{C_{0}} .
$$

Thus, we obtain $\overline{C_{0}}=D$. Let $B_{1}=B \cap D$. For each $x \in A_{1} \subseteq$ $A$, by Lemma 16 , there exists a $y \in B$ such that

$$
M(x, y) \geq H_{M}(A, B)-\varepsilon .
$$

Consequently, $B_{1}$ is a nonempty closed subset of $B$.

For any $x \in A_{1}$, there exists $y \in B_{1}$ such that $M(x, y) \geq$ $H_{M}(A, B)-\varepsilon$, which implies that

$$
M\left(x, B_{1}\right)=\sup _{y \in B_{1}} M(x, y) \geq H_{M}(A, B)-\varepsilon .
$$

Thus we obtain

$$
\rho\left(A_{1}, B_{1}\right)=\inf _{x \in A_{1}} M\left(x, B_{1}\right) \geq H_{M}(A, B)-\varepsilon .
$$

By the definition of $B_{1}$, we can get

$$
M\left(A_{1}, y\right)=\sup _{x \in A_{1}} M(x, y) \geq H_{M}(A, B)-\varepsilon,
$$

for all $y \in B_{1}$. Thus we obtain

$$
\rho\left(B_{1}, A_{1}\right)=\inf _{y \in B_{1}} M\left(A_{1}, y\right) \geq H_{M}(A, B)-\varepsilon .
$$

Consequently, we easily obtain the following inequality:

$$
H_{M}\left(A_{1}, B_{1}\right)=\rho\left(A_{1}, B_{1}\right) \wedge \rho\left(B_{1}, A_{1}\right) \geq H_{M}(A, B)-\varepsilon .
$$

This completes the proof.

Lemma 18 (see [29]). Let $\phi:[0,1] \rightarrow[0,1]$ be a nondecreasing function satisfying the following conditions:

(i) $\phi$ is continuous from the left,

(ii) $\phi^{n}(h) \rightarrow 1 \quad(n \rightarrow \infty)$ for all $h \in(0,1]$, where $\phi^{n}$ denote the $n$th iterative function of $\phi$. Then

(1) for each $h \in(0,1)$, such that $\phi(h)>h$,

(2) $\phi(1)=1$.

Theorem 19. Let $(X, M, *)$ be a complete stationary fuzzy metric space and let $\left\{f_{n}\right\}_{n=1}^{\infty}$ be a sequence of self-mappings of $\mathscr{C} \mathscr{B}(X)$. If there exists a constant $q \in(1,+\infty)$, such that, for each $A_{1}, A_{2} \in \mathscr{C} \mathscr{B}(X)$, and for arbitrary positive integers $i$ and $j, i \neq j$,

$$
\begin{aligned}
& H_{M}\left(f_{i}\left(A_{1}\right), f_{j}\left(A_{2}\right)\right) \\
& \geq q \phi\left(\operatorname { m i n } \left\{H_{M}\left(A_{1}, A_{2}\right), \rho\left(A_{1}, f_{i}\left(A_{1}\right)\right),\right.\right. \\
& \rho\left(A_{2}, f_{j}\left(A_{2}\right)\right), \\
& {\left.\left.\left[\rho\left(A_{2}, f_{i}\left(A_{1}\right)\right)+\rho\left(A_{1}, f_{j}\left(A_{2}\right)\right)\right]^{1 / 2}\right\}\right), }
\end{aligned}
$$

where $\phi$ satisfies the conditions of Lemma 18. Then there exists an $A^{*} \in \mathscr{C} \mathscr{B}(X)$ such that $A^{*} \subseteq f_{i}\left(A^{*}\right)$, for all $i \in \mathbb{N}^{+}$.

Proof. Let $A_{0}, A_{1} \in \mathscr{C} \mathscr{B}(X)$ and $A_{1} \subseteq f_{1}\left(A_{0}\right)$, and $\beta=$ $(1 / q) \in(0,1)$. By Lemma 17 , there exists $A_{2} \in \mathscr{C} \mathscr{B}(X)$, such that $A_{2} \subseteq f_{2}\left(A_{1}\right)$ and

$$
H_{M}\left(A_{1}, A_{2}\right) \geq \beta H_{M}\left(f_{1}\left(A_{0}\right), f_{2}\left(A_{1}\right)\right) .
$$

Again by Lemma 17, we can find $A_{3} \in \mathscr{C} \mathscr{B}(X)$ such that $A_{3} \subseteq$ $f_{3}\left(A_{2}\right)$ and

$$
H_{M}\left(A_{2}, A_{3}\right) \geq \beta H_{M}\left(f_{2}\left(A_{1}\right), f_{3}\left(A_{2}\right)\right)
$$

By induction, we produce a sequence $\left\{A_{n}\right\}$ of points of $\mathscr{C} \mathscr{B}(X)$ such that

$$
\begin{gathered}
A_{n+1} \subseteq f_{n+1}\left(A_{n}\right) \quad n=0,1,2, \ldots \\
H_{M}\left(A_{n+1}, A_{n}\right) \geq \beta H_{M}\left(f_{n+1}\left(A_{n}\right), f_{n}\left(A_{n-1}\right)\right) .
\end{gathered}
$$

Now we prove that $\left\{A_{n}\right\}$ is a Cauchy sequence in $\mathscr{C} \mathscr{B}(X)$. In fact, for arbitrary positive integer $n$, by inequality (38) and formula (41), we have

$$
\begin{aligned}
& H_{M}\left(A_{n}, A_{n+1}\right) \\
& \geq \beta H_{M}\left(f_{n}\left(A_{n-1}\right), f_{n+1}\left(A_{n}\right)\right) \\
& \geq \beta q \phi\left(\operatorname { m i n } \left\{H_{M}\left(A_{n-1}, A_{n}\right),\right.\right. \\
& \rho\left(A_{n-1}, f_{n}\left(A_{n-1}\right)\right), \rho\left(A_{n}, f_{n+1}\left(A_{n}\right)\right), \\
& {\left[\rho\left(A_{n}, f_{n}\left(A_{n-1}\right)\right)\right.} \\
& \left.\left.\left.+\rho\left(A_{n-1}, f_{n+1}\left(A_{n}\right)\right)\right]^{1 / 2}\right\}\right) \\
& =\phi\left(\operatorname { m i n } \left\{H_{M}\left(A_{n-1}, A_{n}\right), \rho\left(A_{n-1}, f_{n}\left(A_{n-1}\right)\right),\right.\right. \\
& \rho\left(A_{n}, f_{n+1}\left(A_{n}\right)\right), \\
& \left.\left.\left[\rho\left(A_{n}, f_{n}\left(A_{n-1}\right)\right)+\rho\left(A_{n-1}, f_{n+1}\left(A_{n}\right)\right)\right]^{1 / 2}\right\}\right) \\
& \geq \phi\left(\operatorname { m i n } \left\{H_{M}\left(A_{n-1}, A_{n}\right), H_{M}\left(A_{n-1}, A_{n}\right),\right.\right. \\
& H_{M}\left(A_{n}, A_{n+1}\right), \\
& \left.\left.\left[1+H_{M}\left(A_{n-1}, A_{n+1}\right)\right]^{1 / 2}\right\}\right) \\
& \left.=\phi\left(\min _{M}\left(A_{n-1}, A_{n}\right), H_{M}\left(A_{n}, A_{n+1}\right)\right\}\right),
\end{aligned}
$$

where $A_{n} \subseteq f_{n}\left(A_{n-1}\right)$, which implies that $\rho\left(A_{n}, f_{n}\left(A_{n-1}\right)\right)=$ 1. then

$$
\text { If } H_{M}\left(A_{n-1}, A_{n}\right) \wedge H_{M}\left(A_{n}, A_{n+1}\right)=H_{M}\left(A_{n}, A_{n+1}\right),
$$

$$
H_{M}\left(A_{n}, A_{n+1}\right) \geq \phi\left(H_{M}\left(A_{n}, A_{n+1}\right)\right) .
$$


From $A_{n+1} \subseteq f_{n+1}\left(A_{n}\right)=\left\{y\right.$ : there exists $x \in A_{n}$ such that $\left.f_{n+1}(x)=y\right\}$, it follows that $H_{M}\left(A_{n}, A_{n+1}\right) \in(0,1]$. Hence, there are two cases.

Case 1. If $H_{M}\left(A_{n}, A_{n+1}\right)=1$, by (2) of Lemma 18 we can get

$$
H_{M}\left(A_{n}, A_{n+1}\right)=H_{M}\left(A_{n-1}, A_{n}\right)=1
$$

that is, $H_{M}\left(A_{n}, A_{n+1}\right) \geq \phi\left(H_{M}\left(A_{n-1}, A_{n}\right)\right)$.

Case 2. If $H_{M}\left(A_{n}, A_{n+1}\right) \in(0,1)$, by (1) of Lemma 18, we can get

$$
H_{M}\left(A_{n}, A_{n+1}\right)<\phi\left(H_{M}\left(A_{n}, A_{n+1}\right)\right)
$$

Obviously, (43) and (45) are contradictory. Hence, we have

$$
H_{M}\left(A_{n-1}, A_{n}\right) \wedge H_{M}\left(A_{n}, A_{n+1}\right)=H_{M}\left(A_{n-1}, A_{n}\right)
$$

that is, $H_{M}\left(A_{n}, A_{n+1}\right) \geq \phi\left(H_{M}\left(A_{n-1}, A_{n}\right)\right)$.

Consequently, we easily obtain the following inequalities:

$$
\begin{aligned}
H_{M}\left(A_{n+1}, A_{n}\right) & \geq \phi\left(H_{M}\left(A_{n}, A_{n-1}\right)\right) \\
& \geq \phi^{2}\left(H_{M}\left(A_{n-1}, A_{n-2}\right)\right) \\
& \geq \cdots \geq \phi^{n}\left(H_{M}\left(A_{1}, A_{0}\right)\right) .
\end{aligned}
$$

Thus, for arbitrary positive integer $p$, we have

$$
\begin{aligned}
H_{M} & \left(A_{n+p}, A_{n}\right) \\
\geq & H_{M}\left(A_{n+p}, A_{n+p-1}\right) \\
& * H_{M}\left(A_{n+p-1}, A_{n+p-2}\right) * \cdots * H_{M}\left(A_{n+1}, A_{n}\right) \\
\geq & \left(\phi^{n+p-1} * \phi^{n+p-2} * \cdots * \phi^{n}\right)\left(H_{M}\left(A_{1}, A_{0}\right)\right) .
\end{aligned}
$$

Since $\phi^{n}(h) \rightarrow 1(n \rightarrow \infty)$, for all $h \in(0,1]$, we get

$$
\begin{aligned}
& \lim _{n \rightarrow \infty} H_{M}\left(A_{n+p}, A_{n}\right) \\
& \quad \geq \lim _{n \rightarrow \infty}\left(\phi^{n+p-1} * \phi^{n+p-2} * \cdots * \phi^{n}\right)\left(H_{M}\left(A_{1}, A_{0}\right)\right) \\
& \quad=1
\end{aligned}
$$

which implies that $\lim _{n \rightarrow \infty} H_{M}\left(A_{n+p}, A_{n}\right)=1$. Hence, $\left\{A_{n}\right\}$ is a Cauchy sequence. In addition, since $(X, M, *)$ is a complete stationary fuzzy metric space, by Theorem 15 , we get that $\left(\mathscr{C} \mathscr{B}(X), H_{M}, *\right)$ is complete. Thus there exists an $A^{*} \in \mathscr{C} \mathscr{B}(X)$ such that $A_{n} \rightarrow A^{*}$ as $n \rightarrow \infty$; that is, $\lim _{n \rightarrow \infty} H_{M}\left(A_{n}, A^{*}\right)=1$.
Next, we show that $A^{*} \subseteq f_{i}\left(A^{*}\right)$, that is, $\rho\left(A^{*}, f_{i}\left(A^{*}\right)\right)=$ 1 , for all $i \in \mathbb{N}^{+}$. In fact, for arbitrary positive integers $i$ and $j$, $i \neq j$, by Proposition 9, we have

$$
\begin{aligned}
\rho\left(A^{*}, f_{i}\left(A^{*}\right)\right) \geq & H_{M}\left(A^{*}, A_{j}\right) * \rho\left(A_{j}, f_{i}\left(A^{*}\right)\right) \\
\geq & H_{M}\left(A^{*}, A_{j}\right) * \rho\left(A_{j}, f_{j}\left(A_{j-1}\right)\right) \\
& * \rho\left(f_{j}\left(A_{j-1}\right), f_{i}\left(A^{*}\right)\right) \\
= & H_{M}\left(A^{*}, A_{j}\right) * \rho\left(f_{j}\left(A_{j-1}\right), f_{i}\left(A^{*}\right)\right) \\
\geq & H_{M}\left(A^{*}, A_{j}\right) * H_{M}\left(f_{j}\left(A_{j-1}\right), f_{i}\left(A^{*}\right)\right) .
\end{aligned}
$$

Moreover, we have

$$
\begin{aligned}
& H_{M}\left(f_{j}\left(A_{j-1}\right), f_{i}\left(A^{*}\right)\right) \\
& \geq q \phi\left(\operatorname { m i n } \left\{H_{M}\left(A_{j-1}, A^{*}\right), \rho\left(A_{j-1}, f_{j}\left(A_{j-1}\right)\right),\right.\right. \\
& \rho\left(A^{*}, f_{i}\left(A^{*}\right)\right), \\
& \left.\left.\left[\rho\left(A^{*}, f_{j}\left(A_{j-1}\right)\right)+\rho\left(A_{j-1}, f_{i}\left(A^{*}\right)\right)\right]^{1 / 2}\right\}\right) \\
& >\phi\left(\operatorname { m i n } \left\{H_{M}\left(A_{j-1}, A^{*}\right), H_{M}\left(A_{j-1}, A_{j}\right),\right.\right. \\
& \rho\left(A^{*}, f_{i}\left(A^{*}\right)\right), \\
& {\left[H_{M}\left(A^{*}, A_{j}\right)+H_{M}\left(A_{j-1}, A^{*}\right)\right.} \\
& \left.\left.\left.* \rho\left(A^{*}, f_{i}\left(A^{*}\right)\right)\right]^{1 / 2}\right\}\right) .
\end{aligned}
$$

Since $\phi$ is continuous from the left and $*$ is a continuous positive $t$-norm, we can obtain

$$
\begin{gathered}
\lim _{j \rightarrow \infty} H_{M}\left(f_{j}\left(A_{j-1}\right), f_{i}\left(A^{*}\right)\right) \\
\geq \lim _{j \rightarrow \infty} \phi\left(\operatorname { m i n } \left\{H_{M}\left(A_{j-1}, A^{*}\right),\right.\right. \\
H_{M}\left(A_{j-1}, A_{j}\right), \rho\left(A^{*}, f_{i}\left(A^{*}\right)\right), \\
{\left[H_{M}\left(A^{*}, A_{j}\right)+H_{M}\left(A_{j-1}, A^{*}\right)\right.} \\
\left.\left.\left.* \rho\left(A^{*}, f_{i}\left(A^{*}\right)\right)\right]^{1 / 2}\right\}\right) \\
=\phi\left(\operatorname { m i n } \left\{1,1, \rho\left(A^{*}, f_{i}\left(A^{*}\right)\right),\right.\right. \\
\left.\left.\left[1+1 * \rho\left(A^{*}, f_{i}\left(A^{*}\right)\right)\right]^{1 / 2}\right\}\right) \\
=\phi\left(\rho\left(A^{*}, f_{i}\left(A^{*}\right)\right)\right) .
\end{gathered}
$$


Consequently, we conclude that

$$
\begin{aligned}
\rho\left(A^{*}, f_{i}\left(A^{*}\right)\right) \geq & \lim _{j \rightarrow \infty} H_{M}\left(A^{*}, A_{j}\right) \\
& * \lim _{j \rightarrow \infty} H_{M}\left(f_{j}\left(A_{j-1}\right), f_{i}\left(A^{*}\right)\right) \\
\geq & 1 * \phi\left(\rho\left(A^{*}, f_{i}\left(A^{*}\right)\right)\right) \\
& =\phi\left(\rho\left(A^{*}, f_{i}\left(A^{*}\right)\right)\right) ;
\end{aligned}
$$

that is, $\rho\left(A^{*}, f_{i}\left(A^{*}\right)\right)=1$. By (1) of Proposition 9, we obtain $A^{*} \subseteq f_{i}\left(A^{*}\right)$, for all $i \in \mathbb{N}^{+}$.

Corollary 20. Let $(X, M, *)$ be a complete stationary fuzzy metric space and let $f$ be a self-mapping of $\mathscr{C} \mathscr{B}(X)$. If there exists a constant $q \in(1,+\infty)$, such that, for each $A_{1}, A_{2} \in$ $\mathscr{C} \mathscr{B}(X)$,

$$
\begin{gathered}
H_{M}\left(f\left(A_{1}\right), f\left(A_{2}\right)\right) \\
\geq q \phi\left(\operatorname { m i n } \left\{H_{M}\left(A_{1}, A_{2}\right), \rho\left(A_{1}, f\left(A_{1}\right)\right), \rho\left(A_{2}, f\left(A_{2}\right)\right),\right.\right. \\
\left.\left.\left[\rho\left(A_{2}, f\left(A_{1}\right)\right)+\rho\left(A_{1}, f\left(A_{2}\right)\right)\right]^{1 / 2}\right\}\right),
\end{gathered}
$$

where $\phi$ satisfies the conditions of Lemma 18, then there exists an $A^{*} \in \mathscr{C} \mathscr{B}(X)$ such that $A^{*} \subseteq f\left(A^{*}\right)$.

Proof. In fact, we can define a sequence of fuzzy selfmappings of $\mathscr{C} \mathscr{C}(X)$ as $f_{i}=f$, for $i=1,2, \ldots$. Thus, this result is a special case of Theorem 19.

Theorem 21. Let $(X, M, *)$ be a complete stationary fuzzy metric space and let $\left\{f_{n}\right\}_{n=1}^{\infty}$ be a sequence of self-mappings of $\mathscr{C} \mathscr{B}(X)$. If there exists a constant $q \in(1,+\infty)$, such that, for each $A_{1}, A_{2} \in \mathscr{C} \mathscr{B}(X)$, and for arbitrary positive integers $i$ and $j, i \neq j$,

$$
\begin{aligned}
& H_{M}\left(f_{i}\left(A_{1}\right), f_{j}\left(A_{2}\right)\right) \\
& \geq q \phi\left(H_{M}\left(A_{1}, A_{2}\right), \rho\left(A_{1}, f_{i}\left(A_{1}\right)\right), \rho\left(A_{2}, f_{j}\left(A_{2}\right)\right),\right. \\
& \left.\quad \rho\left(A_{2}, f_{i}\left(A_{1}\right)\right), \rho\left(A_{1}, f_{j}\left(A_{2}\right)\right)\right),
\end{aligned}
$$

where $\phi\left(h_{1}, h_{2}, h_{3}, h_{4}, h_{5}\right):(0,1]^{5} \rightarrow[0,1]$ is nondecreasing and continuous from the left for each variable, let $r^{n}(h)=$ $\phi(h, h, h, a, b)$, where $(a, b) \in\{(h * h, 1),(1, h * h)\}$. If

$$
r^{n}(h) \longrightarrow 1 \text { as } n \longrightarrow \infty, \quad \forall h \in(0,1]
$$

where ${ }^{n}$ denotes the nth iterative function of $r$, then there exists an $A^{*} \in \mathscr{C} \mathscr{B}(X)$ such that $A^{*} \subseteq f_{i}\left(A^{*}\right)$, for all $i \in \mathbb{N}^{+}$.

Proof. Let $A_{0}, A_{1} \in \mathscr{C} \mathscr{B}(X)$ and $A_{1} \subseteq f_{1}\left(A_{0}\right)$, and $\beta=$ $(1 / q) \in(0,1)$. By Lemma 17 , there exists $A_{2} \in \mathscr{C} \mathscr{B}(X)$, such that $A_{2} \subseteq f_{2}\left(A_{1}\right)$ and

$$
H_{M}\left(A_{1}, A_{2}\right) \geq \beta H_{M}\left(f_{1}\left(A_{0}\right), f_{2}\left(A_{1}\right)\right) .
$$

Again by Lemma 17, we can find $A_{3} \in \mathscr{C} \mathscr{B}(X)$ such that $A_{3} \subseteq$ $f_{3}\left(A_{2}\right)$ and

$$
H_{M}\left(A_{2}, A_{3}\right) \geq \beta H_{M}\left(f_{2}\left(A_{1}\right), f_{3}\left(A_{2}\right)\right) .
$$

By induction, we produce a sequence $\left\{A_{n}\right\}$ of points of $\mathscr{C} \mathscr{B}(X)$ such that

$$
\begin{gathered}
A_{n+1} \subseteq f_{n+1}\left(A_{n}\right), \quad n=0,1,2, \ldots \\
H_{M}\left(A_{n+1}, A_{n}\right) \geq \beta H_{M}\left(f_{n+1}\left(A_{n}\right), f_{n}\left(A_{n-1}\right)\right) .
\end{gathered}
$$

Now we prove that $\left\{A_{n}\right\}$ is a Cauchy sequence in $\mathscr{C} \mathscr{B}(X)$. In fact, for arbitrary positive integer $n$, by inequality (55) and formula (59), we have

$$
\begin{aligned}
& H_{M}\left(A_{n}, A_{n+1}\right) \\
& \geq \beta H_{M}\left(f_{n}\left(A_{n-1}\right), f_{n+1}\left(A_{n}\right)\right) \\
& \geq \beta q \phi\left(H_{M}\left(A_{n-1}, A_{n}\right), \rho\left(A_{n-1}, f_{n}\left(A_{n-1}\right)\right),\right. \\
& \quad \rho\left(A_{n}, f_{n+1}\left(A_{n}\right)\right), \rho\left(A_{n-1}, f_{n+1}\left(A_{n}\right)\right), \\
& \left.\quad \rho\left(A_{n}, f_{n}\left(A_{n-1}\right)\right)\right) \\
& =\phi\left(H_{M}\left(A_{n-1}, A_{n}\right), \rho\left(A_{n-1}, f_{n}\left(A_{n-1}\right)\right),\right. \\
& \rho\left(A_{n}, f_{n+1}\left(A_{n}\right)\right), \rho\left(A_{n-1}, f_{n+1}\left(A_{n}\right)\right), \\
& \left.\rho\left(A_{n}, f_{n}\left(A_{n-1}\right)\right)\right) \\
& \geq \phi\left(H_{M}\left(A_{n-1}, A_{n}\right), H_{M}\left(A_{n-1}, A_{n}\right), H_{M}\left(A_{n}, A_{n+1}\right),\right. \\
& \left.H_{M}\left(A_{n-1}, A_{n}\right) * H_{M}\left(A_{n}, A_{n+1}\right), 1\right),
\end{aligned}
$$

where $A_{n} \subseteq f_{n}\left(A_{n-1}\right)$, which implies that $\rho\left(A_{n}, f_{n}\left(A_{n-1}\right)\right)=$ 1.

If $H_{M}\left(A_{n-1}, A_{n}\right) \wedge H_{M}\left(A_{n}, A_{n+1}\right)=H_{M}\left(A_{n}, A_{n+1}\right)$, then

$$
H_{M}\left(A_{n}, A_{n+1}\right) \geq r\left(H_{M}\left(A_{n}, A_{n+1}\right)\right) .
$$

From

$$
\begin{aligned}
& A_{n+1} \subseteq f_{n+1}\left(A_{n}\right) \\
& =\left\{y: \text { there exists an } x \in A_{n} \text { such that } f_{n+1}(x)=y\right\},
\end{aligned}
$$

we get $H_{M}\left(A_{n}, A_{n+1}\right) \in(0,1]$. Hence, there are two cases.

Case 1. If $H_{M}\left(A_{n}, A_{n+1}\right)=1$, by (2) of Lemma 18, we can get

$$
H_{M}\left(A_{n}, A_{n+1}\right)=H_{M}\left(A_{n-1}, A_{n}\right)=1 ;
$$

that is, $H_{M}\left(A_{n}, A_{n+1}\right) \geq r\left(H_{M}\left(A_{n-1}, A_{n}\right)\right)$.

Case 2. If $H_{M}\left(A_{n}, A_{n+1}\right) \in(0,1)$, by (1) of Lemma 18, we can get

$$
H_{M}\left(A_{n}, A_{n+1}\right)<r\left(H_{M}\left(A_{n}, A_{n+1}\right)\right) .
$$


Obviously, (61) and (64) are contradictory. Hence, we have

$$
H_{M}\left(A_{n-1}, A_{n}\right) \wedge H_{M}\left(A_{n}, A_{n+1}\right)=H_{M}\left(A_{n-1}, A_{n}\right)
$$

that is, $H_{M}\left(A_{n}, A_{n+1}\right) \geq r\left(H_{M}\left(A_{n-1}, A_{n}\right)\right)$.

Consequently, we easily obtain the following relations:

$$
\begin{aligned}
H_{M}\left(A_{n+1}, A_{n}\right) & \geq r\left(H_{M}\left(A_{n}, A_{n-1}\right)\right) \\
& \geq r^{2}\left(H_{M}\left(A_{n-1}, A_{n-2}\right)\right) \\
& \geq \cdots \geq r^{n}\left(H_{M}\left(A_{1}, A_{0}\right)\right) .
\end{aligned}
$$

Thus, for arbitrary positive integer $p$, we have

$$
\begin{aligned}
H_{M}\left(A_{n+p}, A_{n}\right) \geq & H_{M}\left(A_{n+p}, A_{n+p-1}\right) \\
& * H_{M}\left(A_{n+p-1}, A_{n+p-2}\right) \\
& * \cdots * H_{M}\left(A_{n+1}, A_{n}\right) \\
\geq & \left(r^{n+p-1} * r^{n+p-2} * \cdots * r^{n}\right) \\
& \times\left(H_{M}\left(A_{1}, A_{0}\right)\right) .
\end{aligned}
$$

$\operatorname{By} r^{n}(h) \rightarrow 1(n \rightarrow \infty)$, for all $h \in(0,1]$, and continuity of $*$, we can get

$$
\begin{aligned}
& \lim _{n \rightarrow \infty} H_{M}\left(A_{n+p}, A_{n}\right) \\
& \quad \geq \lim _{n \rightarrow \infty}\left(r^{n+p-1} * r^{n+p-2} * \cdots * r^{n}\right)\left(H_{M}\left(A_{1}, A_{0}\right)\right)=1,
\end{aligned}
$$

which implies that $\lim _{n \rightarrow \infty} H_{M}\left(A_{n+p}, A_{n}\right)=1$. Hence, we get $\left\{A_{n}\right\} \subseteq \mathscr{C} \mathscr{B}(X)$ that is a Cauchy sequence. In addition, since $(X, M, *)$ is a complete stationary fuzzy metric space, by Theorem 15, we have that $\left(\mathscr{C} \mathscr{B}(X), H_{M}\right.$,*) is complete. Thus there exists an $A^{*} \in \mathscr{C} \mathscr{B}(X)$ such that $A_{n} \rightarrow A^{*}$ as $n \rightarrow \infty$; that is, $\lim _{n \rightarrow \infty} H_{M}\left(A_{n}, A^{*}\right)=1$.

Next, we show that $A^{*} \subseteq f_{i}\left(A^{*}\right)$, that is, $\rho\left(A^{*}, f_{i}\left(A^{*}\right)\right)=$ 1 , for all $i \in \mathbb{N}^{+}$. In fact, for arbitrary positive integers $i$ and $j$, $i \neq j$, by Proposition 9, we have

$$
\begin{aligned}
\rho\left(A^{*}, f_{i}\left(A^{*}\right)\right) \geq & H_{M}\left(A^{*}, A_{j}\right) * \rho\left(A_{j}, f_{i}\left(A^{*}\right)\right) \\
\geq & H_{M}\left(A^{*}, A_{j}\right) * \rho\left(A_{j}, f_{j}\left(A_{j-1}\right)\right) \\
& * \rho\left(f_{j}\left(A_{j-1}\right), f_{i}\left(A^{*}\right)\right) \\
= & H_{M}\left(A^{*}, A_{j}\right) * \rho\left(f_{j}\left(A_{j-1}\right), f_{i}\left(A^{*}\right)\right) \\
\geq & H_{M}\left(A^{*}, A_{j}\right) * H_{M}\left(f_{j}\left(A_{j-1}\right), f_{i}\left(A^{*}\right)\right) .
\end{aligned}
$$

Moreover, we have

$$
\begin{aligned}
& H_{M}\left(f_{j}\left(A_{j-1}\right), f_{i}\left(A^{*}\right)\right) \\
& \geq q \phi\left(H_{M}\left(A_{j-1}, A^{*}\right), \rho\left(A_{j-1}, f_{j}\left(A_{j-1}\right)\right),\right. \\
& \quad \rho\left(A^{*}, f_{i}\left(A^{*}\right)\right), \rho\left(A_{j-1}, f_{i}\left(A^{*}\right)\right), \\
& \left.\quad \rho\left(A^{*}, f_{j}\left(A_{j-1}\right)\right)\right) \\
& >\phi\left(H_{M}\left(A_{j-1}, A^{*}\right), \rho\left(A_{j-1}, f_{j}\left(A_{j-1}\right)\right), \rho\left(A^{*}, f_{i}\left(A^{*}\right)\right),\right. \\
& \left.\quad \rho\left(A_{j-1}, f_{i}\left(A^{*}\right)\right), \rho\left(A^{*}, f_{j}\left(A_{j-1}\right)\right)\right) \\
& \geq \phi\left(H_{M}\left(A_{j-1}, A^{*}\right), H_{M}\left(A_{j-1}, A_{j}\right), \rho\left(A^{*}, f_{i}\left(A^{*}\right)\right),\right. \\
& \left.H_{M}\left(A_{j-1}, A^{*}\right) * \rho\left(A^{*}, f_{i}\left(A^{*}\right)\right), H_{M}\left(A^{*}, A_{j}\right)\right) .
\end{aligned}
$$

Since $\phi$ is continuous from the left and $*$ is a continuous positive $t$-norm, hence, we can obtain

$$
\begin{aligned}
& \lim _{j \rightarrow \infty} H_{M}\left(f_{j}\left(A_{j-1}\right), f_{i}\left(A^{*}\right)\right) \\
& \geq \lim _{j \rightarrow \infty} \phi\left(H_{M}\left(A_{j-1}, A^{*}\right), H_{M}\left(A_{j-1}, A_{j}\right), \rho\left(A^{*}, f_{i}\left(A^{*}\right)\right),\right. \\
& \left.\quad H_{M}\left(A_{j-1}, A^{*}\right) * \rho\left(A^{*}, f_{i}\left(A^{*}\right)\right), H_{M}\left(A^{*}, A_{j}\right)\right) \\
& =\phi\left(1,1, \rho\left(A^{*}, f_{i}\left(A^{*}\right)\right), \rho\left(A^{*}, f_{i}\left(A^{*}\right)\right), 1\right) \\
& \geq \phi\left(\rho\left(A^{*}, f_{i}\left(A^{*}\right)\right), \rho\left(A^{*}, f_{i}\left(A^{*}\right)\right), \rho\left(A^{*}, f_{i}\left(A^{*}\right)\right),\right. \\
& \left.\quad \rho\left(A^{*}, f_{i}\left(A^{*}\right)\right) * \rho\left(A^{*}, f_{i}\left(A^{*}\right)\right), 1\right) \\
& =r\left(\rho\left(A^{*}, f_{i}\left(A^{*}\right)\right)\right) .
\end{aligned}
$$

Consequently, we conclude that

$$
\begin{aligned}
\rho\left(A^{*}, f_{i}\left(A^{*}\right)\right) \geq & \lim _{j \rightarrow \infty} H_{M}\left(A^{*}, A_{j}\right) \\
& * \lim _{j \rightarrow \infty} H_{M}\left(f_{j}\left(A_{j-1}\right), f_{i}\left(A^{*}\right)\right) \\
\geq & 1 * r\left(\rho\left(A^{*}, f_{i}\left(A^{*}\right)\right)\right) \\
& =r\left(\rho\left(A^{*}, f_{i}\left(A^{*}\right)\right)\right) ;
\end{aligned}
$$

that is, $\rho\left(A^{*}, f_{i}\left(A^{*}\right)\right)=1$. By (1) of Proposition 9, we obtain $A^{*} \subseteq f_{i}\left(A^{*}\right)$, for all $i \in \mathbb{N}^{+}$.

Corollary 22. Let $(X, M, *)$ be a complete stationary fuzzy metric space and let $f$ be a self-mapping of $\mathscr{C} \mathscr{B}(X)$. If there exists a constant $q \in(1,+\infty)$, such that, for each $A_{1}, A_{2} \in$ $\mathscr{C} \mathscr{B}(X)$,

$$
\begin{aligned}
& H_{M}\left(f\left(A_{1}\right), f\left(A_{2}\right)\right) \\
& \geq q \phi\left(H_{M}\left(A_{1}, A_{2}\right), \rho\left(A_{1}, f\left(A_{1}\right)\right), \rho\left(A_{2}, f\left(A_{2}\right)\right),\right. \\
& \left.\quad \rho\left(A_{2}, f\left(A_{1}\right)\right), \rho\left(A_{1}, f\left(A_{2}\right)\right)\right),
\end{aligned}
$$


where $\phi\left(h_{1}, h_{2}, h_{3}, h_{4}, h_{5}\right):(0,1]^{5} \rightarrow[0,1]$ is nondecreasing and continuous from the left for each variable, let $r^{n}(h)=$ $\phi(h, h, h, a, b)$, where $(a, b) \in\{(h * h, 1),(1, h * h)\}$. If

$$
r^{n}(h) \longrightarrow 1 \quad \text { as } n \longrightarrow \infty, \quad \forall h \in(0,1]
$$

where $r^{n}$ denotes the $n$th iterative function of $r$, then there exists an $A^{*} \in \mathscr{C} \mathscr{B}(X)$ such that $A^{*} \subseteq f\left(A^{*}\right)$.

Proof. In fact, we can define a sequence of fuzzy selfmappings of $\mathscr{C} \mathscr{C}(X)$ as $f_{i}=f$, for $i=1,2, \ldots$ Thus, this result is a special case of Theorem 21 .

Theorem 23. Let $(X, M, *)$ be a complete stationary fuzzy metric space and let $\left\{f_{n}\right\}_{n=1}^{\infty}$ be a sequence of self-mappings of $\mathscr{C} \mathscr{B}(X)$. If there exists a constant $q \in(1,+\infty)$, such that, for each $A_{1}, A_{2} \in \mathscr{C} \mathscr{B}(X)$, and, for arbitrary positive integers $i$ and $j, i \neq j$,

$$
H_{M}\left(f_{i}\left(A_{1}\right), f_{j}\left(A_{2}\right)\right) \geq q \phi\left(H_{M}\left(A_{1}, A_{2}\right)\right),
$$

where $\phi$ satisfies the conditions of Lemma 18, then there exists an $A^{*} \in \mathscr{C} \mathscr{B}(X)$ such that $A^{*} \subseteq f_{i}\left(A^{*}\right)$, for all $i \in \mathbb{N}^{+}$.

Proof. Let $A_{0}, A_{1} \in \mathscr{C} \mathscr{B}(X)$ and $A_{1} \subseteq f_{1}\left(A_{0}\right)$, and $\beta=$ $(1 / q) \in(0,1)$. By Lemma 17 , there exists $A_{2} \in \mathscr{C} \mathscr{B}(X)$, such that $A_{2} \subseteq f_{2}\left(A_{1}\right)$ and

$$
H_{M}\left(A_{1}, A_{2}\right) \geq \beta H_{M}\left(f_{1}\left(A_{0}\right), f_{2}\left(A_{1}\right)\right) .
$$

Again by Lemma 17, we can find $A_{3} \in \mathscr{C} \mathscr{B}(X)$ such that $A_{3} \subseteq$ $f_{3}\left(A_{2}\right)$ and

$$
H_{M}\left(A_{2}, A_{3}\right) \geq \beta H_{M}\left(f_{2}\left(A_{1}\right), f_{3}\left(A_{2}\right)\right) .
$$

By induction, we produce a sequence $\left\{A_{n}\right\}$ of points of $\mathscr{C} \mathscr{B}(X)$ such that

$$
\begin{gathered}
A_{n+1} \subseteq f_{n+1}\left(A_{n}\right), \quad n=0,1,2, \ldots, \\
H_{M}\left(A_{n+1}, A_{n}\right) \geq \beta H_{M}\left(f_{n+1}\left(A_{n}\right), f_{n}\left(A_{n-1}\right)\right) .
\end{gathered}
$$

Now we prove that $\left\{A_{n}\right\}$ is a Cauchy sequence in $\mathscr{C} \mathscr{B}(X)$. In fact, for arbitrary positive integer $n$, by inequality (75) and formula (78), we have

$$
\begin{aligned}
H_{M}\left(A_{n+1}, A_{n}\right) & \geq \beta H_{M}\left(f_{n+1}\left(A_{n}\right), f_{n}\left(A_{n-1}\right)\right) \\
& \geq \beta q \phi\left(H_{M}\left(A_{n}, A_{n-1}\right)\right) \\
& =\phi\left(H_{M}\left(A_{n}, A_{n-1}\right)\right) .
\end{aligned}
$$

Thus, from inequality (79), we easily obtain the following relations:

$$
\begin{aligned}
H_{M}\left(A_{n+1}, A_{n}\right) & \geq \phi\left(H_{M}\left(A_{n}, A_{n-1}\right)\right) \\
& \geq \phi^{2}\left(H_{M}\left(A_{n}, A_{n-1}\right)\right) \\
& \geq \cdots \geq \phi^{n}\left(H_{M}\left(A_{1}, A_{0}\right)\right) .
\end{aligned}
$$

Furthermore, for arbitrary positive integers $n$ and $p$, we get that

$$
\begin{aligned}
H_{M}\left(A_{n+p}, A_{n}\right) \geq & H_{M}\left(A_{n+p}, A_{n+p-1}\right) \\
& * H_{M}\left(A_{n+p-1}, A_{n+p-2}\right) \\
& * \cdots * H_{M}\left(A_{n+1}, A_{n}\right) \\
\geq & \left(\phi^{n+p-1} * \phi^{n+p-2} * \cdots * \phi^{n}\right) \\
& \times\left(H_{M}\left(A_{1}, A_{0}\right)\right) .
\end{aligned}
$$

Since for arbitrary $h \in(0,1), \phi^{n}(h) \rightarrow 1(n \rightarrow \infty)$, and by continuity of $*$, we have

$$
H_{M}\left(A_{n+p}, A_{n}\right) \longrightarrow 1 \quad(n \longrightarrow \infty) ;
$$

that is, $\left\{A_{n}\right\}$ is a Cauchy sequence in $\mathscr{C} \mathscr{B}(X)$. By Theorem 15, $\mathscr{C} \mathscr{B}(X)$ is complete since $X$ is complete. Consequently, there exists $A^{*} \in \mathscr{C} \mathscr{B}(X)$ such that $A_{n} \rightarrow A^{*}(n \rightarrow \infty)$; that is, $\lim _{n \rightarrow \infty} H_{M}\left(A_{n}, A^{*}\right)=1$.

Next, we show that $A^{*} \subseteq f_{i}\left(A^{*}\right)$; that is, $\rho\left(A^{*}, f_{i}\left(A^{*}\right)\right)=$ 1 , for all $i \in \mathbb{N}^{+}$. In fact, for arbitrary positive integers $i$ and $j$, $i \neq j$, by Proposition 9 we have

$$
\begin{aligned}
\rho\left(A^{*}, f_{i}\left(A^{*}\right)\right) \geq & H_{M}\left(A^{*}, A_{j}\right) * \rho\left(A_{j}, f_{i}\left(A^{*}\right)\right) \\
\geq & H_{M}\left(A^{*}, A_{j}\right) * \rho\left(A_{j}, f_{j}\left(A_{j-1}\right)\right) \\
& * \rho\left(f_{j}\left(A_{j-1}\right), f_{i}\left(A^{*}\right)\right) \\
= & H_{M}\left(A^{*}, A_{j}\right) * \rho\left(f_{j}\left(A_{j-1}\right), f_{i}\left(A^{*}\right)\right) \\
\geq & H_{M}\left(A^{*}, A_{j}\right) * H_{M}\left(f_{j}\left(A_{j-1}\right), f_{i}\left(A^{*}\right)\right) .
\end{aligned}
$$

Moreover, we have

$$
\begin{aligned}
H_{M}\left(f_{j}\left(A_{j-1}\right), f_{i}\left(A^{*}\right)\right) & \geq q \phi\left(H_{M}\left(A_{j-1}, A^{*}\right)\right) \\
& >\phi\left(H_{M}\left(A_{j-1}, A^{*}\right)\right) .
\end{aligned}
$$

Consequently, we get

$$
\rho\left(A^{*}, f_{i}\left(A^{*}\right)\right)>H_{M}\left(A^{*}, A_{j}\right) * \phi\left(H_{M}\left(A_{j-1}, A^{*}\right)\right) .
$$

Since $\phi$ is continuous from the left and $*$ is a continuous positive $t$-norm, hence, we can obtain

$$
\begin{aligned}
\rho\left(A^{*}, f_{i}\left(A^{*}\right)\right) \geq & \lim _{j \rightarrow \infty} H_{M}\left(A^{*}, A_{j}\right) \\
& * \phi\left(\lim _{j \rightarrow \infty} H_{M}\left(A_{j-1}, A^{*}\right)\right)=1 \\
& * \phi(1)=1 ;
\end{aligned}
$$

that is, $\rho\left(A^{*}, f_{i}\left(A^{*}\right)\right)=1$. By (1) of Proposition 9, we obtain $A^{*} \subseteq f_{i}\left(A^{*}\right)$, for all $i \in \mathbb{N}^{+}$. 
Corollary 24. Let $(X, M, *)$ be a complete stationary fuzzy metric space and let $f$ be a self-mapping of $\mathscr{C} \mathscr{B}(X)$. If there exists a constant $q \in(1,+\infty)$, such that, for each $A_{1}, A_{2} \in$ $\mathscr{C} \mathscr{B}(X)$,

$$
H_{M}\left(f\left(A_{1}\right), f\left(A_{2}\right)\right) \geq q \phi\left(H_{M}\left(A_{1}, A_{2}\right)\right),
$$

where $\phi$ satisfies the conditions of Lemma 18, then there exists an $A^{*} \in \mathscr{C} \mathscr{B}(X)$ such that $A^{*} \subseteq f\left(A^{*}\right)$.

Proof. In fact, we can define a sequence of fuzzy selfmappings of $\mathscr{C} \mathscr{B}(X)$ as $f_{i}=f$, for $i=1,2, \ldots$ Thus, this result is a special case of Theorem 23 .

Example 25. Let $\left(\mathscr{C} \mathscr{B}(X), H_{M_{d}}, \cdot\right)$ be a stationary fuzzy metric space, where $X=[-1,1]$, and $H_{M_{d}}$ is the same as in Example 13. Then, $\left(\mathscr{C} \mathscr{B}(X), H_{M_{d}}, \cdot\right)$ is a complete stationary fuzzy metric space.

Now, define $\phi:[0,1] \rightarrow[0,1]$ as $\phi(x)=\sqrt{x}$, and let $\left\{f_{n}\right\}_{n=1}^{\infty}$ be a sequence of self-mappings of $\mathscr{C} \mathscr{B}(X)$ as

$$
f_{n}(A)=\frac{1}{2^{n}} A, \quad \text { for any } A \in \mathscr{C} \mathscr{B}(X) .
$$

For arbitrary positive integers $i$ and $j$, without loss of generality, suppose $i<j$. For each $A_{1}, A_{2} \in \mathscr{C} \mathscr{B}(X)$, by a routine calculation, we have

$$
\begin{aligned}
H_{M_{d}}\left(f_{i}\left(A_{1}\right), f_{j}\left(A_{2}\right)\right) & =H_{M_{d}}\left(\frac{1}{2^{i}} A_{1}, \frac{1}{2^{j}} A_{2}\right) \\
& =2^{i} H_{M_{d}}\left(A_{1}, \frac{1}{2^{j-i}} A_{2}\right) \\
& \geq 2^{i} H_{M_{d}}\left(A_{1}, A_{2}\right) \\
& \geq 2 \phi\left(H_{M_{d}}\left(A_{1}, A_{2}\right)\right) .
\end{aligned}
$$

Therefore, by Theorem 23, we assert that the sequence of selfmappings $\left\{f_{n}\right\}_{n=1}^{\infty}$ has a common fixed point $A^{*}$ in $\mathscr{C} \mathscr{B}(X)$. In fact, it is easy to check that $A^{*}=\{0\}$.

\section{Conclusions}

In this paper, we have established the completeness of $\mathscr{C} \mathscr{B}(X)$ with respect to the completeness of the stationary fuzzy metric space $X$. We also present some common fixed point theorems for the self-mapping of stationary fuzzy metric space $\mathscr{C} \mathscr{B}(X)$ under some $\phi$-contraction conditions.

Several possible applications of our results may be suggested. We briefly mention some of them. Fuzzy fixed point theory can be used in existence and continuity theorems for dynamical systems with some vague parameters [45-47]. More specifically in the field of qualitative behavior, these may be used demonstrating the existence of solutions of the fuzzy differential equation [25] and fuzzy integral equation, and so forth. In addition, the recent work of Veeramani [48] on best approximation in fuzzy metric spaces could constitute the start of a theory for which the Hausdorff fuzzy metric must play an important role. So we hope our results would provide a mathematical background to ongoing work in the problems of those related fields.

\section{Acknowledgments}

This work was supported by the National Natural Science Foundation of China (Grant no. 11201512) and the Natural Science Foundation Project of CQ CSTC (cstc2012jjA00001).

\section{References}

[1] J.-P. Aubin and I. Ekeland, Applied Nonlinear Analysis, John Wiley \& Sons Inc., New York, NY, USA, 1984.

[2] D. Azé, J.-N. Corvellec, and R. E. Lucchetti, "Variational pairs and applications to stability in nonsmooth analysis," Nonlinear Analysis A: Theory, Methods \& Applications, vol. 49, no. 5, pp. 643-670, 2002.

[3] G. Beer, Topologies on Closed and Closed Convex Sets, vol. 268, Kluwer Academic Publishers, Dordrecht, The Netherlands, 1993.

[4] J. Gutiérrez García, S. Romaguera, and M. Sanchis, "An identification theorem for the completion of the Hausdorff fuzzy metric," Fuzzy Sets and Systems, vol. 227, pp. 96-106, 2013.

[5] F. Hausdorff, Set Theory, Chelsea, New York, NY, USA, 1957.

[6] N. van Hop, "Solving fuzzy (stochastic) linear programming problems using superiority and inferiority measures," Information Sciences, vol. 177, no. 9, pp. 1977-1991, 2007.

[7] N. van Hop, "Solving linear programming problems under fuzziness and randomness environment using attainment values," Information Sciences, vol. 177, no. 14, pp. 2971-2984, 2007.

[8] S. Y. Joo and Y. K. Kim, "Topological properties on the space of fuzzy sets," Journal of Mathematical Analysis and Applications, vol. 246, no. 2, pp. 576-590, 2000.

[9] Y. K. Kim, "Compactness and convexity on the space of fuzzy sets," Journal of Mathematical Analysis and Applications, vol. 264, no. 1, pp. 122-132, 2001.

[10] Y. K. Kim, "Compactness and convexity on the space of fuzzy sets. II," Nonlinear Analysis A: Theory, Methods \& Applications, vol. 57, no. 5-6, pp. 639-653, 2004.

[11] D. Qiu and W. Zhang, "The strongest t-norm for fuzzy metric spaces," Kybernetika, vol. 49, pp. 141-148, 2013.

[12] L. A. Zadeh, "Fuzzy sets," Information and Computation, vol. 8, pp. 338-353, 1965.

[13] S. S. Chang, Y. J. Cho, B. S. Lee, J. S. Jung, and S. M. Kang, "Coincidence point theorems and minimization theorems in fuzzy metric spaces," Fuzzy Sets and Systems, vol. 88, no. 1, pp. 119-127, 1997.

[14] A. George and P. Veeramani, "On some results in fuzzy metric spaces,” Fuzzy Sets and Systems, vol. 64, no. 3, pp. 395-399, 1994.

[15] V. Gregori and S. Romaguera, "On completion of fuzzy metric spaces," Fuzzy Sets and Systems, vol. 130, no. 3, pp. 399-404, 2002.

[16] O. Kaleva and S. Seikkala, "On fuzzy metric spaces," Fuzzy Sets and Systems, vol. 12, no. 3, pp. 215-229, 1984.

[17] I. Kramosil and J. Michálek, "Fuzzy metrics and statistical metric spaces," Kybernetika, vol. 11, no. 5, pp. 336-344, 1975.

[18] G. Matheron, Random Sets and Integral Geometry, John Wiley \& Sons, New York, NY, USA, 1975.

[19] J. Rodríguez-López, S. Romaguera, and J. M. Sánchez-Álvarez, "The Hausdorff fuzzy quasi-metric," Fuzzy Sets and Systems, vol. 161, no. 8, pp. 1078-1096, 2010.

[20] J. Rodríguez-López and S. Romaguera, "The Hausdorff fuzzy metric on compact sets," Fuzzy Sets and Systems, vol. 147, no. 2, pp. 273-283, 2004. 
[21] A. Azam and I. Beg, "Common fuzzy fixed points for fuzzy mappings," Fixed Point Theory and Applications, vol. 2013, article 14, 11 pages, 2013.

[22] M. Abbas, M. Imdad, and D. Gopal, " $\psi$-weak contractions in fuzzy metric spaces," Iranian Journal of Fuzzy Systems, vol. 8, no. 5, pp. 141-148, 2011.

[23] I. Beg, C. Vetro, D. Gopal, and M. Imdad, “( $\phi, \psi)$-weak contractions in intuitionistic fuzzy metric spaces," Journal of Intelligent and Fuzzy Systems, 2013.

[24] D. Gopal, M. Imdad, C. Vetro, and M. Hasan, "Fixed point theory for cyclic weak $\phi$-contraction infuzzy metric spaces," Journal of Nonlinear Analysis and Application, vol. 2012, Article ID IDjnaa-00110, 11 pages, 2012.

[25] O. Kaleva, "Fuzzy differential equations," Fuzzy Sets and Systems, vol. 24, no. 3, pp. 301-317, 1987.

[26] D. Miheţ, "The fixed point method for fuzzy stability of the Jensen functional equation," Fuzzy Sets and Systems, vol. 160, no. 11, pp. 1663-1667, 2009.

[27] D. Miheţ, "A class of contractions in fuzzy metric spaces," Fuzzy Sets and Systems, vol. 161, no. 8, pp. 1131-1137, 2010.

[28] S. B. Nadler, Jr., "Multi-valued contraction mappings," Pacific Journal of Mathematics, vol. 30, pp. 475-488, 1969.

[29] D. Qiu, L. Shu, and J. Guan, "Common fixed point theorems for fuzzy mappings under $\Phi$-contraction condition," Chaos, Solitons and Fractals, vol. 41, no. 1, pp. 360-367, 2009.

[30] M. Tanveer, M. Imdad, D. Gopal, and D. K. Patel, "Common fixed point theorems in modified intuitionisticfuzzy metric spaces with common property," Fixed Point Theory and Applications, vol. 2012, article 36, 2012.

[31] H. Adibi, Y. J. Cho, D. O’Regan, and R. Saadati, “Common fixed point theorems in $L$-fuzzy metric spaces," Applied Mathematics and Computation, vol. 182, no. 1, pp. 820-828, 2006.

[32] Y. J. Cho and N. Petrot, "Existence theorems for fixed fuzzy points with closed $\alpha$-cut sets in complete metric spaces," Korean Mathematical Society, vol. 26, no. 1, pp. 115-124, 2011.

[33] B. M. Ghil and Y. K. Kim, "Continuity of functions defined on the space of fuzzy sets," Information Sciences, vol. 157, pp. 155165, 2003.

[34] V. Gregori and A. Sapena, "On fixed-point theorems in fuzzy metric spaces," Fuzzy Sets and Systems, vol. 125, no. 2, pp. 245252, 2002.

[35] O. Kaleva, "On the convergence of fuzzy sets," Fuzzy Sets and Systems, vol. 17, no. 1, pp. 53-65, 1985.

[36] M. L. Puri and D. A. Ralescu, "Fuzzy random variables," Journal of Mathematical Analysis and Applications, vol. 114, no. 2, pp. 409-422, 1986.

[37] D. Qiu and L. Shu, "Supremum metric on the space of fuzzy sets and common fixed point theorems for fuzzy mappings," Information Sciences, vol. 178, no. 18, pp. 3595-3604, 2008.

[38] W. Zhang, D. Qiu, Z. Li, and G. Xiong, "Common fixed point theorems in a new fuzzy metric space," Journal of Applied Mathematics, vol. 2012, Article ID 890678, 18 pages, 2012.

[39] E. P. Klement, R. Mesiar, and E. Pap, Triangular Norms, vol. 8, Kluwer Academic Publishers, Dordrecht, The Netherlands, 2000.

[40] V. Gregori and S. Romaguera, "Characterizing completable fuzzy metric spaces," Fuzzy Sets and Systems, vol. 144, no. 3, pp. 411-420, 2004.

[41] D. Qiu, W. Zhang, and C. Li, "Extension of a class of decomposable measures using fuzzy pseudometrics," Fuzzy Sets and Systems, vol. 222, pp. 33-44, 2013.
[42] D. Qiu, C. X. Lu, L. Wang, and W. Zhang, "On the hyperspace of bounded closed sets under a generalizedHausdorff stationary fuzzy metric, submited to Bull," The Korean Mathematical Society, 2013.

[43] D. Qiu, W. Zhang, and C. Li, "On decomposable measures constructed by using stationary fuzzy pseudo-ultrametrics," International Journal of General Systems, vol. 42, no. 4, pp. 395-404, 2013.

[44] R. Engelking, General Topology, PWN-Polish Scientific Publishers, Warsaw, Poland, 1977.

[45] W. Fei, "Existence and uniqueness of solution for fuzzy random differential equations with non-Lipschitz coefficients," Information Sciences, vol. 177, no. 20, pp. 4329-4337, 2007.

[46] M. T. Mizukoshi, L. C. Barros, Y. Chalco-Cano, H. RománFlores, and R. C. Bassanezi, "Fuzzy differential equations and the extension principle," Information Sciences, vol. 177, no. 17, pp. 3627-3635, 2007.

[47] G. Papaschinopoulos, G. Stefanidou, and P. Efraimidis, "Existence, uniqueness and asymptotic behavior of the solutions of a fuzzy differential equation with piecewise constant argument," Information Sciences, vol. 177, no. 18, pp. 3855-3870, 2007.

[48] P. Veeramani, "Best approximation in fuzzy metric spaces," Journal of Fuzzy Mathematics, vol. 9, no. 1, pp. 75-80, 2001. 


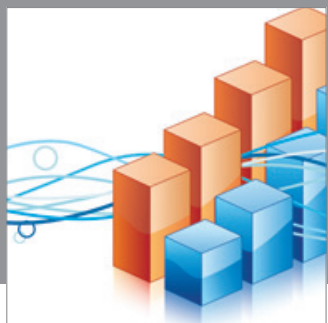

Advances in

Operations Research

mansans

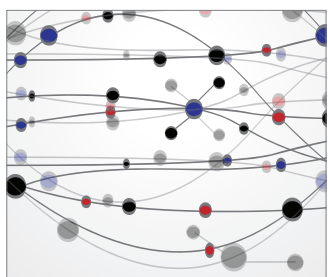

The Scientific World Journal
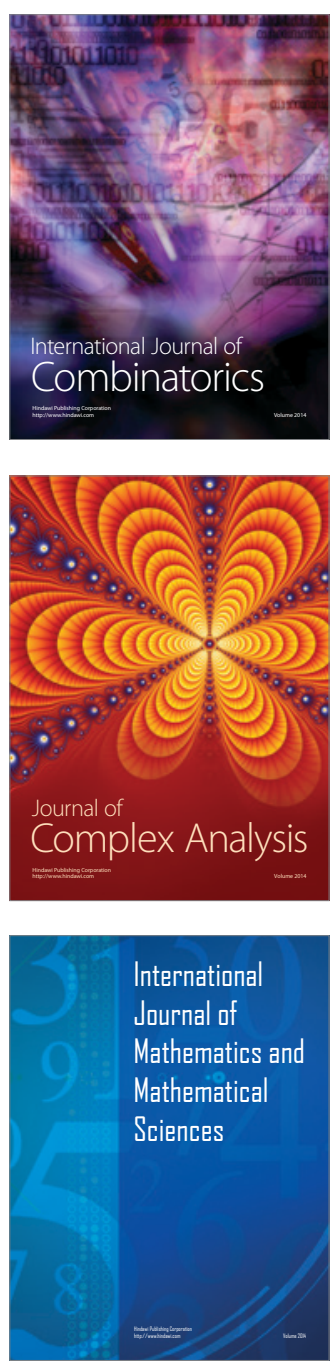
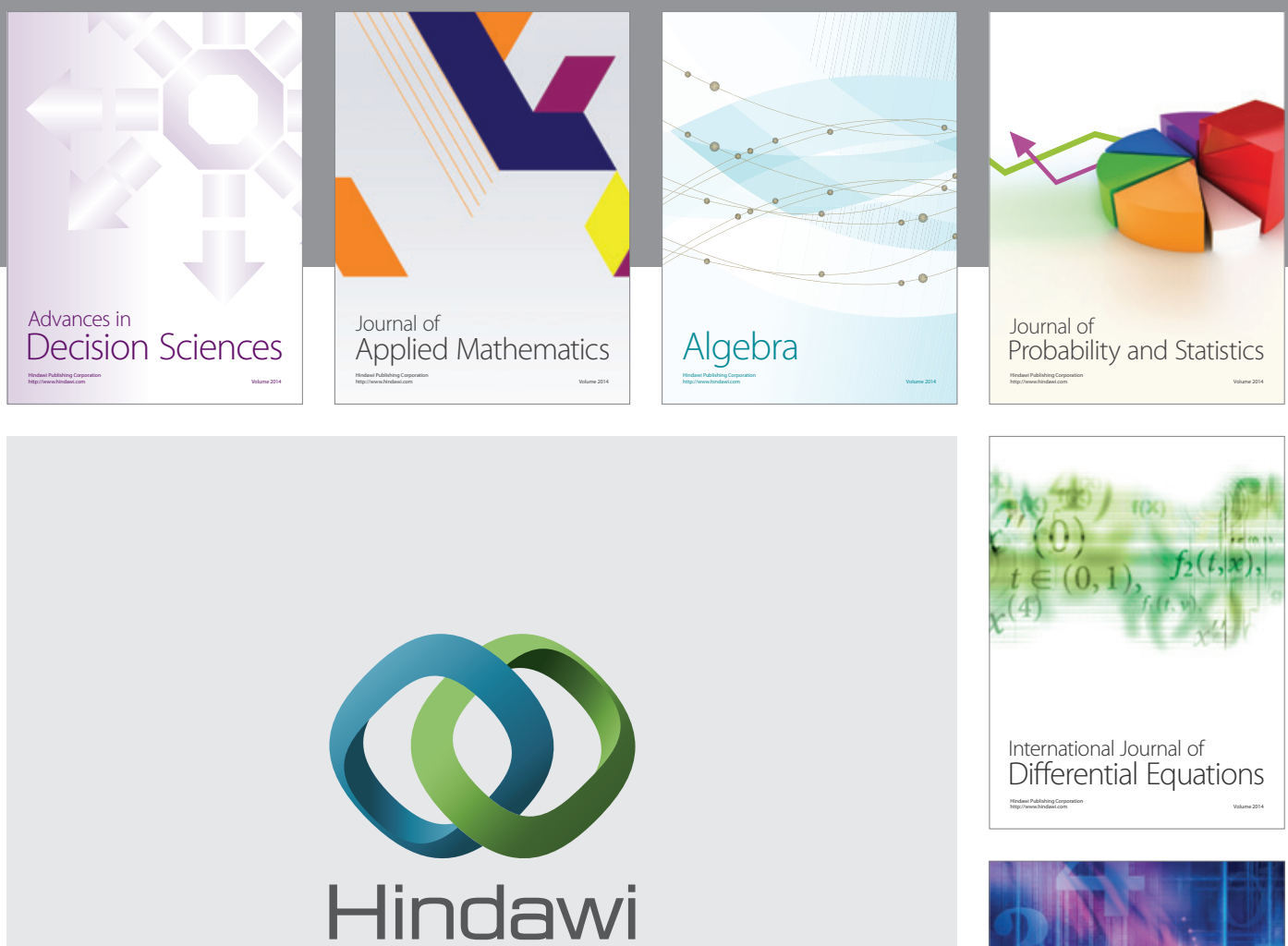

Submit your manuscripts at http://www.hindawi.com
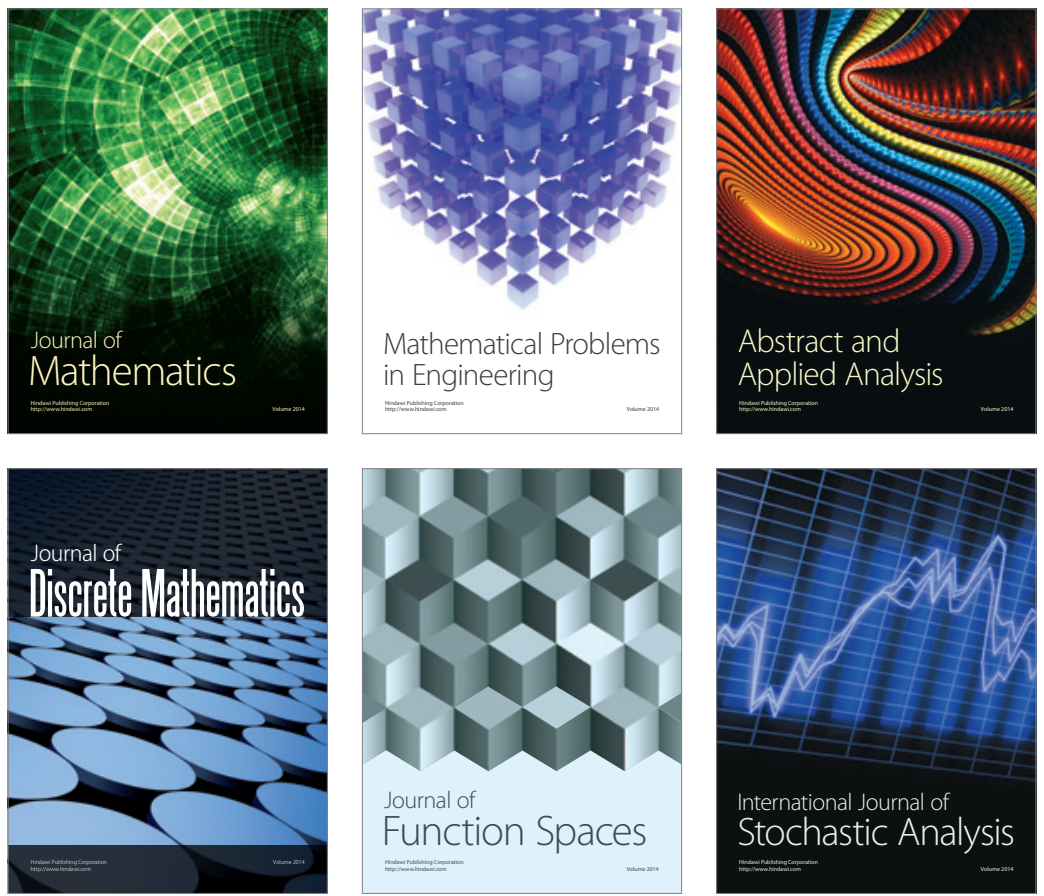

Journal of

Function Spaces

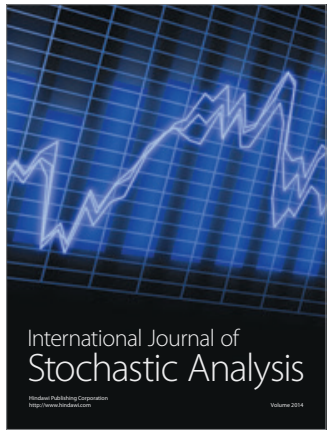

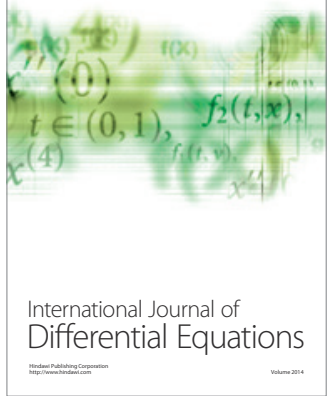
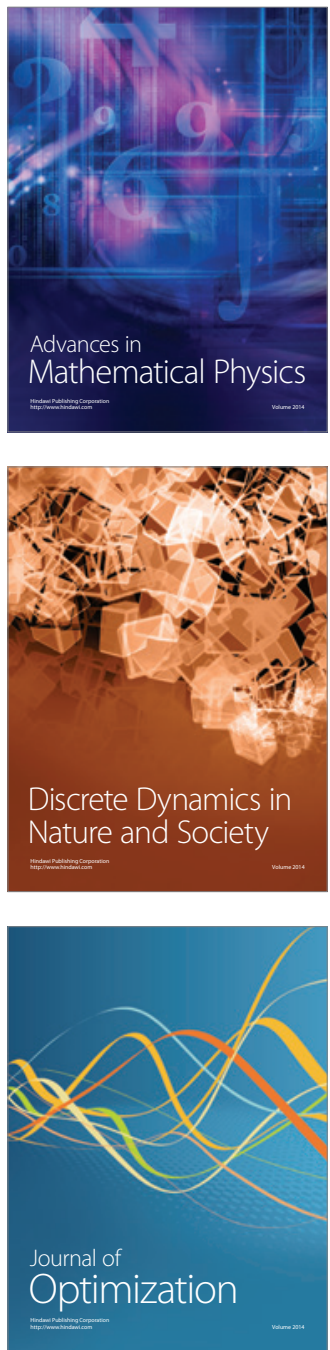